\title{
Automorphisms of Riemann-Cartan Manifolds with Semi-Symmetric Connection
}

\author{
V.I. Panzhensky \\ Penza State Pedagogical University \\ 37 Lermontov Str., Penza 440206, Russia \\ E-mail: kaf_geom@pnzgu.ru
}

Received December 13, 2012, revised January 15, 2014

It is proved that the maximum dimension of the Lie group of automorphisms of a Riemann-Cartan manifold $(M, g, \tilde{\nabla})$ is $\frac{n(n-1)}{2}+1$, where $M$ is a smooth $n$-dimensional manifold, $g$ is a Riemannian or semi-Riemannian metric on $M, \tilde{\nabla}$ is a semi-symmetric connection.

Key words: Riemann-Cartan manifolds, auto_morphisms, semi-symmetric connection.

Mathematics Subject Classification 2010: 53B50.

1. A smooth $n$-dimensional manifold $M$ with a semi-Riemannian metric $g$ and a linear metric connection $\tilde{\nabla}$ with torsion is called a Riemann-Cartan manifold [1]. The connection $\tilde{\nabla}$ can be represented as $\tilde{\nabla}=\hat{\nabla}+\frac{1}{2} \tilde{S}$, where $\hat{\nabla}$ is an associated symmetric connection and $\tilde{S}$ is a torsion tensor of the connection $\tilde{\nabla}$. On the other hand, $\tilde{\nabla}=\nabla+\tilde{T}$, where $\nabla$ is the Levi-Civita connection of the metric $g$ and $\tilde{T}$ is the deformation tensor of the connection $\nabla$. The covariant deformation tensor $T$ determined by the equality $T(X, Y, Z)=g(\tilde{T}(X, Y), Z)$ is skew-symmetric with respect to the last two arguments because of the covariant constancy of the metric tensor $g$ in the connection $\tilde{\nabla}, \tilde{\nabla} g=0$. Thus, the Riemann-Cartan structure $(g, \tilde{\nabla})$ is unambiguously defined by setting a pair of tensor fields $(g, T)$, namely, a metric tensor and a deformation tensor, the first of which is symmetric with respect to its arguments, and the second one is skew-symmetric in the last two arguments. We also note that the deformation tensor is defined unambiguously by the torsion tensor and vice versa, while the symmetric part $\hat{\nabla}$ of the connection $\tilde{\nabla}$ coincides with the Levi-Civita connection $\nabla$ if and only if the tensor T is skewsymmetric with respect to its arguments [2]. In this case, $T=\frac{1}{2} S$, where $S$ is a covariant tensor of torsion, and the connection $\tilde{\nabla}$ is called skew-symmetric. If

$$
S(X, Y, Z)=g(X, Z) \Theta(Y)-g(Y, Z) \Theta(X),
$$

where $\Theta=\frac{1}{n-1} \operatorname{trace} \tilde{S}$, then the connection $\tilde{\nabla}$ is called semi-symmetric. 
2. A diffeomorphism $\varphi: M \rightarrow M$ is said to be an automorphism of the Riemann-Cartan manifold if $g$ and $\tilde{\nabla}$ remain invariant under $\varphi$. Since $\tilde{\nabla}=\nabla+\tilde{T}$ and the invariance of $\nabla$ follows from the invariance of $g$ [3], then the connection $\tilde{\nabla}$ is invariant if and only if the deformation tensor $\tilde{T}$ is invariant, which is equivalent to the invariance of the covariant deformation tensor $T$. Thus the set of all automorphisms of the Riemann-Cartan manifold $(M, g, \tilde{\nabla})$ either coincides with the Lie group of isometries of semi-Riemannian manifold $(M, g)$ or is its closed Lie subgroup which leaves the tensor field $T$ invariant and, therefore, it has the dimension $r \leq \frac{n(n+1)}{2}$.

Theorem 1. The dimension of the Lie group of automorphisms of an n-dimensional Riemann-Cartan manifold with semi-symmetric connection is not larger than $\frac{n(n-1)}{2}+1$.

P r o o f. Let $\mathbb{G}$ be an $r$-dimensional Lie group of automorphisms of an $n$-dimensional Riemann-Cartan manifold $M$. The stationary subgroup of a point $x_{0} \in M$ induces the isotropy group $\mathbb{G}_{0}$ in the tangent space $\mathbb{E}=T_{x_{0}} M$. The vector space $\mathbb{E}=\mathbb{E}_{p, q}^{n}$ is an $n$-dimensional Euclidean space $(p=n, q=0)$ or a semiEuclidean space with $(p, q)$-signature $(+\ldots+,-\ldots-)$. The value of a torsion tensor field $\tilde{S}$ at $x_{0} \in M$ is a nonzero tensor in $\left(\mathbb{E}^{*} \wedge \mathbb{E}^{*}\right) \otimes \mathbb{E}$. Let us consider $\tilde{S}$ as a skew-symmetric mapping $\mathbb{E} \times \mathbb{E} \rightarrow \mathbb{E}$. The isotropy group $\mathbb{G}_{0}$ is a subgroup of the group of orthogonal or pseudo-orthogonal transformations of $\mathbb{E}$. Let $\xi$ be an element of the Lie algebra of the Lie group of (pseudo) orthogonal transformations of $\mathbb{E}$, and $\varphi_{t}=\exp t \xi$ be a one-parameter subgroup of transformations generated by $\xi$. Then $\xi$ belongs to the Lie algebra $g_{0}$ of the Lie group $\mathbb{G}_{0}$ if and only if the tensor $\tilde{S}$ remains invariant under $\varphi_{t}$, i.e.,

$$
\tilde{S}\left(\varphi_{t} u, \varphi_{t} v\right)=\varphi_{t} \tilde{S}(u, v) .
$$

Differentiating (1) with respect to $t$ at $t=0$, we get

$$
\tilde{S}(\xi u, v)+\tilde{S}(u, \xi v)=\xi \tilde{S}(u, v) .
$$

Let $\left(e_{1}, \ldots, e_{n}\right)$ be a (pseudo) orthonormal basis in $\mathbb{E}$, and $S_{i j}^{k}$ and $\xi_{i}^{j}$ be components $\tilde{S}$ and $\xi$ in this basis. Then (2) has the form

$$
S_{s j}^{k} \xi_{i}^{s}+S_{i s}^{k} \xi_{j}^{s}-S_{i j}^{r} \xi_{r}^{k}=0
$$

or

$$
\left(S_{s j}^{k} \delta_{i}^{r}+S_{i s}^{k} \delta_{j}^{r}-S_{i j}^{r} \delta_{s}^{k}\right) \xi_{r}^{s}=0,
$$

where $\delta_{i}^{j}$ is the Kronecker symbol.

Let now the connection $\tilde{\nabla}$ be semi-symmetric. Then

$$
S_{i j}^{k}=\frac{1}{n-1}\left(\delta_{i}^{k} \eta_{j}-\delta_{j}^{k} \eta_{i}\right),
$$


where $\eta_{j}=S_{* j}^{*}$ denote the components of a 1 -form in $\mathbb{E}$. Substituting (5) into (4), we get

$$
\left(\delta_{s}^{k} \eta_{j} \delta_{i}^{r}-\delta_{j}^{k} \eta_{s} \delta_{i}^{r}+\delta_{i}^{k} \eta_{s} \delta_{j}^{r}-\delta_{s}^{k} \eta_{i} \delta_{j}^{r}-\delta_{i}^{r} \eta_{j} \delta_{s}^{k}+\delta_{j}^{r} \eta_{i} \delta_{s}^{k}\right) \xi_{r}^{s}=0
$$

or

$$
\left(\delta_{i}^{k} \delta_{j}^{r}-\delta_{j}^{k} \delta_{i}^{r}\right) \eta_{s} \xi_{r}^{s}=0 .
$$

If $\mathbb{E}$ is Euclidean, then the Lie algebra of the Lie group of orthogonal transformations of $\mathbb{E}$ consists of skew-symmetric matrices, i.e., $\xi_{r}^{s}=-\xi_{s}^{r}$. If $\mathbb{E}=\mathbb{E}_{p, q}^{n}$ is semi-Euclidean, then the matrix $\xi$ has the form

$$
\left(\begin{array}{ll}
A & B \\
C & D
\end{array}\right)
$$

where $A$ is a $p \times p$ skew-symmetric matrix, $D$ is a $q \times q$ skew-symmetric matrix, and $C=B^{T}$ is the matrix transposed to $B$. In any case, $\xi_{1}^{1}=\xi_{2}^{2}=\ldots=\xi_{n}^{n}=0$ and $\xi_{r}^{s}= \pm \xi_{s}^{r}$. Thus we get a system of linear equations (6) with respect to $\frac{\left(n^{2}-n\right)}{2}$ unknown $\xi_{r}^{s}$. Let us prove that this system contains at least $n-1$ linearly independent equations. Actually, (6) can be written as follows:

$$
\left(\delta_{i}^{k} \delta_{j}^{r}-\delta_{j}^{k} \delta_{i}^{r}\right) \eta_{s} \xi_{r}^{s}+\left(\delta_{i}^{k} \delta_{j}^{s}-\delta_{j}^{k} \delta_{i}^{s}\right) \eta_{r} \xi_{s}^{r}=0 .
$$

As the 1-form $\eta$ is nonzero, then at least one of its coordinates is not zero. Let $\eta_{s} \neq 0$ for some $s$. We consider the subsystem consisting of $n-1$ equations with the indices $i=k=s, j=1, \ldots, n ; j \neq s$. The subsystem takes the form

$$
\ldots+\left(\delta_{s}^{s} \delta_{j}^{r}-\delta_{j}^{s} \delta_{s}^{r}\right) \eta_{s} \xi_{r}^{s}+\left(\delta_{s}^{s} \delta_{j}^{s}-\delta_{j}^{s} \delta_{s}^{s}\right) \eta_{r} \xi_{s}^{r}+\ldots=0
$$

or

$$
\ldots+\delta_{j}^{r} \eta_{s} \xi_{r}^{s}+\ldots=0 \quad(r=1, \ldots, n ; r \neq s),
$$

and it is linearly independent because the matrix $\left(\delta_{j}^{r} \eta_{s}\right)$ is obviously nondegenerate. Therefore the dimension of the isotropy group $\mathbb{G}_{0}$ is not larger than $\frac{\left(n^{2}-n\right)}{2}-(n-1)$, and the dimension of the group of all automorphisms is not larger than $\frac{\left(n^{2}-n\right)}{2}-(n-1)+n=\frac{n(n-1)}{2}+1$.

3. Theorem 2. The maximum dimension of the Lie group of automorphisms of an n-dimensional Riemann-Cartan manifold with semi-symmetric connection is equal to $\frac{n(n-1)}{2}+1$.

$\mathrm{P}$ r o o f. To prove the theorem, it is enough to give an example of an $n$-dimensional Riemann-Cartan manifold with automorphism group of dimension 
$\frac{n(n-1)}{2}+1$. Let us consider the semi-Riemannian space $M^{n}, n \geq 3$, with the metric form

$$
d s^{2}=d x^{1^{2}}+e^{2 H x^{1}}\left(\varepsilon_{2} d x^{2^{2}}+\ldots+\varepsilon_{n} d x^{n 2}\right),
$$

where $\varepsilon_{\alpha}= \pm 1, \alpha=2, \ldots, n, H=$ const. Calculating the curvature tensor of this space, we verify the validity of the equality

$$
R_{i j k l}=-H^{2}\left(g_{i l} g_{j k}-g_{i k} g_{j l}\right) .
$$

It follow then that $M^{n}$ has a constant sectional curvature $k=-H^{2}$. Consequently, the isometry group of this space has the maximum dimension $\frac{n(n+1)}{2}$. Let us consider a closed subgroup of the group containing all isometries which leave invariant a single vector field orthogonal to the semi-Euclidean subspace $\mathbb{E}^{n-1}, x^{1}=$ const, with the metric form

$$
d \sigma^{2}=\varepsilon_{2} d x^{2^{2}}+\ldots+\varepsilon_{n} d x^{n 2} .
$$

Basic operators of this subgroup are

$$
\partial_{\alpha}, \quad-\varepsilon_{\alpha} x^{\beta} \partial_{\alpha}+\varepsilon_{\beta} x^{\alpha} \partial_{\beta}, \quad-\frac{1}{H} \partial_{1}+x^{\alpha} \partial_{\alpha}, \quad \alpha<\beta, \alpha, \beta=2, \ldots, n .
$$

In (11), the first $\frac{n(n-1)}{2}$ vector fields are basic operators of the Lie group of isometries of the space $\mathbb{E}^{n-1}$ with metric (10), and the last vector field is defined by the invariance of the metrics (9) and a single vector field orthogonal to $\mathbb{E}^{n-1}$ with respect to the last vector field. The condition of the invariance of the deformation tensor $T_{i j k}$ with respect to the vector field $\mathrm{X}=\xi^{p} \partial_{p}$ takes the form

$$
\xi^{p} \partial_{p} T_{i j k}+\partial_{i} \xi^{p} T_{p j k}+\partial_{j} \xi^{p} T_{i p k}+\partial_{k} \xi^{p} T_{i j p}=0
$$

To find the deformation tensor $T_{i j k}$, which is invariant with respect to the group of operators (11), it is necessary to write a corresponding differential equation (12) for each vector field (11) and then integrate the obtained system of partial differential equations. Fortunately, this task becomes much more simplified if the connection is semi-symmetric. For the semi-symmetric connection we have

$$
T_{i j k}=\frac{1}{n-1}\left(g_{i k} \eta_{j}-g_{i j} \eta_{k}\right) .
$$

Hence the invariance of $T_{i j k}$ leads to the invariance of $\eta_{j}=T_{* j}^{*}$ and vice versa. That is why, it is enough to integrate the equations of the invariance of $\eta$

$$
\xi^{p} \partial_{p} \eta_{j}+\partial_{j} \xi^{p} \eta_{p}=0
$$


and restore $T_{i j k}$ according to (13). As a result, we get $\eta=c d x^{1}, c=$ const, and

$$
T=a e^{2 H x^{1}} \sum_{\alpha} \varepsilon_{\alpha} d x^{\alpha} \otimes d x^{\alpha} \wedge d x^{1}, \quad a=\text { const. }
$$

Thus we have an example of the $n$-dimensional Riemann-Cartan manifold $(n \geq 3)$ with semi-symmetric connection whose automorphism group has dimension $\frac{n(n-1)}{2}+1$. The metric tensor and deformation tensor of the manifold are defined by (9) and (15), respectively, and basic operators are defined by (11).

4. In Einstein's general theory of relativity (GTR) and its generalizations, the basic subject is a four-dimensional semi-Riemannian manifold of signature $(+---)$ which we call a space-time manifold $M^{4}$. The metric form for $M^{4}$, given in the proof of Theorem 2, can be rewritten in the following way:

$$
d s^{2}=d x^{0^{2}}-e^{2 H x^{0}}\left(d x^{1^{2}}+d x^{2^{2}}+d x^{3^{2}}\right), x^{0}=c t .
$$

It is well known (see, e.g., [4]) that this metric is the solution of the Einstein equation with $\Lambda$-term

$$
R_{i j}-\frac{1}{2} R g_{i j}=\frac{8 \pi G}{c^{4}} T_{i j}+\Lambda g_{i j},
$$

which defines the stationary model of the Universe. Contrary to Friedmann's solutions, this solution has no singularity. Nevertheless, the metrics of the stationary model describes an expansion of the Universe occurring without a bound in time both in the past and in the future. The Hubble constant $H$ (redshift of spectral lines) is then unchanged during the Universe evolution, and the cosmological constant is $\Lambda=\frac{3 H}{c^{2}}$. A spatial section $\left(x^{0}=c t=\right.$ const $)$ is a Euclidean space, i.e., in this model the world is flat and without matter, therefore the theory of the stationary Universe cannot be applied to the Universe with matter. But endowing a stationary model with additional structures, as is done, for example, in the theory of compensations, may allow solving some problems existing in the framework of the theory of a stationary Universe. Cartan was the first to draw the physicists' attention to the need that torsion be taken into account for generalizations of GTR ((1922), see, e.g., [5]). In one of the attempts to create the uniform gravitation and electromagnetic theory (1928), Einstein used a connection with torsion but without curvature (connection of absolute parallelism). Subsequently in the Einstein-Cartan theory, the torsion is introduced to geometrize the matter spin density, the spin is represented by a covector $\eta$ defining the torsion. It means that the connection $\tilde{\nabla}$ must be semi-symmetric. Moreover, for this connection to have the maximum symmetry number, the deformation tensor of the connection must have the following form according to formula (15):

$$
T=a e^{2 H x^{0}} \sum_{\alpha=1}^{3} d x^{\alpha} \otimes d x^{\alpha} \wedge d x^{0}, \quad a=\text { const. }
$$


By integrating the invariance equation of deformation tensor (12) with respect to the isometry group (11), we obtain the general solution

$$
T=a e^{2 H x^{0}} \sum_{\alpha=1}^{3} d x^{\alpha} \otimes d x^{\alpha} \wedge d x^{0}+b e^{3 H x^{0}} d x^{1} \wedge d x^{2} \wedge d x^{3},
$$

where $a, b=$ const. Alongside with the "spin" part $T_{a}$, there is the skewsymmetric part $T_{b}$ defining the torsion of the spatial section $x^{0}=$ const which may not be a spin.

5. In the spatial section $\left(x^{0}=\right.$ const $)$, which is the Euclidean space $E^{3}$, the torsion tensor takes the form

$$
S=s d x^{1} \wedge d x^{2} \wedge d x^{3},
$$

where a constant $s$ is called a space torsion. In [6], we introduced the concepts of the scalar torsion and volume torsion for three-dimensional spaces with skewsymmetric torsion. We denote the fundamental form of torsion by $\Omega=s_{123}$. $d x^{1} \wedge d x^{2} \wedge d x^{3}$, and the volume form by $\Omega_{0}=\sqrt{g} d x^{1} \wedge d x^{2} \wedge d x^{3}, g=\operatorname{det}\left\|g_{\alpha \beta}\right\|$. Then the following integrals are well defined for any bounded domain $D$ :

$$
v=\int_{D} \Omega, v_{0}=\int_{D} \Omega_{0}
$$

We call the relation $v_{k}=\frac{v}{v_{0}}$ the volume torsion, and the relation $s_{k}=\frac{s_{123}}{\sqrt{g}}$ the scalar torsion. The volume torsion is a functional defined on the set of bounded domains $D$, and the scalar torsion is a function. If the domain of integration is contracted to a point, then the volume torsion converges to the scalar torsion at this point. In our case, the volume torsion $v_{k}$ coincides with the scalar torsion $s_{k}$ and is equal to the torsion $s$ of $E^{3}$.

6. We study the law of parallel translation of vectors in the connection $\tilde{\nabla}$ with skew-symmetric torsion (19). We find coefficients $\tilde{\Gamma}_{i j}^{k}$ of the connection $\tilde{\nabla}$ :

$$
\tilde{\Gamma}_{12}^{3}=\tilde{\Gamma}_{23}^{1}=\tilde{\Gamma}_{31}^{2}=-\tilde{\Gamma}_{21}^{3}=-\tilde{\Gamma}_{32}^{1}=-\tilde{\Gamma}_{13}^{2}=s,
$$

and all other coefficients are zeros. The equations of parallel translation

$$
\frac{d v^{k}}{d t}+\tilde{\Gamma}_{i j}^{k} \frac{d x^{i}}{d t} v^{j}=0
$$

of a vector $v^{k}=v^{k}(t)$ along a curve $x^{k}=x^{k}(t)$ take the form

$$
\frac{d v^{1}}{d t}+s\left(\frac{d x^{2}}{d t} v^{3}-\frac{d x^{3}}{d t} v^{2}\right)=0
$$




$$
\begin{aligned}
& \frac{d v^{2}}{d t}+s\left(\frac{d x^{3}}{d t} v^{1}-\frac{d x^{1}}{d t} v^{3}\right)=0 \\
& \frac{d v^{3}}{d t}+s\left(\frac{d x^{1}}{d t} v^{2}-\frac{d x^{2}}{d t} v^{1}\right)=0
\end{aligned}
$$

Let us study in more detail a parallel translation, for example, of the vector $v(1,0,0)$ along the curve $x^{1}=0, x^{2}=0, x^{3}=t$, i.e., along the $x^{3}$-axis of the Cartesian coordinate system in $E^{3}$. In this case, equations (22) have the form

$$
\frac{d v^{1}}{d t}-s v^{2}=0, \quad \frac{d v^{2}}{d t}+s v^{1}=0, \quad \frac{d v^{3}}{d t}=0 .
$$

Integrating (23), we find its general solution

$$
\begin{gathered}
v^{1}=\sqrt{c_{1}^{2}+c_{2}^{2}} \cos \left(s t-\varphi_{0}\right), \\
v^{2}=-\sqrt{c_{1}^{2}+c_{2}^{2}} \sin \left(s t-\varphi_{0}\right), \\
v^{3}=c_{3},
\end{gathered}
$$

where $\varphi_{0}=\operatorname{arctg} \frac{c_{2}}{c_{1}}$.

From the initial data it follows that $c_{1}=1, c_{2}=c_{3}=0$. Therefore, under parallel translation, the end-point of $v$ makes a helix curve

$$
\vec{r}=\vec{r}\{\cos (s t), \sin (s t), t\}
$$

lying on the straight helicoid which is swept by the $x^{1}$-axis under its parallel translation along the axis $x^{3}$. In this case, the torsion $s$ determines the angular rotation speed of $v$ under its parallel translation along the straight line.

\section{References}

[1] I.A.Gordeyeva, V.I.Panzhensky, and S.E. Stepanov, Riemann-Cartan Manifolds. — J. Math. Sci. 169 (2009), No. 3, 342-361.

[2] K. Yano and S. Bochner, Curvature and Betti Numbers. Ann. Math. Stud. No. 32, Princeton Univ. Pres., Princeton, 1953.

[3] L.P. Eisenhart, Riemannian Geometry. Princeton Univ. Press 1968.

[4] Y.B. Zeldovich and N.D. Novikov, The Structure and Evolution of the Universe. Nauka, Moscow, 1975. (Russian)

[5] S.E. Stepanov and I.A. Gordeyeva, Pseudo-killing and Pseudoharmonic Vector Fields on a Riemann-Cartan Manifold. - Math. Notes 87 (2010), 248-257.

[6] V.I. Panzhensky, Maximally Movable Riemannian Spaces with Torsion. - Math. Notes 85 (2009), No. 5, 720-723. 